\title{
Une intellectuelle, auteure et mécène parmi d'autres : Marguerite de Valois (1553-1615)
}

\section{Éliane VIENNOT}

\section{(2) OpenEdition}

1 Journals

\section{Édition électronique}

URL : https://journals.openedition.org/clio/137

DOI : $10.4000 /$ clio. 137

ISSN : 1777-5299

Éditeur

Belin

Édition imprimée

Date de publication : 1 avril 2001

Pagination : 125-134

ISBN : 2-85816-577-7

ISSN : 1252-7017

Référence électronique

Éliane VIENNOT, « Une intellectuelle, auteure et mécène parmi d'autres : Marguerite de Valois (1553-1615) », Clio. Histoire, femmes et sociétés [En ligne], 13 | 2001, mis en ligne le 19 juin 2006, consulté le 24 avril 2022. URL : http://journals.openedition.org/clio/137 ; DOI : https://doi.org/ 10.4000/clio. 137

Ce document a été généré automatiquement le 24 avril 2022.

Tous droits réservés 


\title{
Une intellectuelle, auteure et mécène parmi d'autres : Marguerite de Valois (1553-1615)
}

\author{
Éliane VIENNOT
}

1 Marguerite de Valois, dernière fille d'Henri II et de Catherine de Médicis, est arrivée à l'âge adulte à un moment relativement propice pour l'accès des femmes à la culture et à la publication. Elle est née en effet un siècle et demi après la fracassante arrivée sur la scène intellectuelle de la première auteure des temps modernes, Christine de Pizan, et une soixantaine d'années après l'impression du premier écrit féminin (Proba Falconia, Paris, 1490). Bien d'autres femmes, en ce milieu du xvie siècle, avaient alors fait imprimer leurs écrits: Marguerite d'Autriche, Anne de France, Claudine Scève, Marguerite de Navarre, Marie d'Ennetières [ou Dentière], Hélisenne de Crenne, Pernette du Guillet, les sœurs Seymour, Antoinette de Luynes..., sans compter quelques anonymes. Dans les vingt années suivantes, la liste s'était encore allongée : Louise Labé, Marguerite de Cambis, Anne de Marquets, Antoinette Peronnet, Marie de Costeblanche, Georgette de Montenay ${ }^{1} . .$.

2 Pourtant, les obstacles mis à cette "venue à l'écriture » des femmes et à son inscription dans le marché éditorial (en plein essor) n'étaient pas minces. D'abord en raison des difficultés rencontrées dans l'accès à l'instruction: les filles des milieux populaires pouvaient certes fréquenter les écoles paroissiales, et celles des milieux supérieurs recevoir les leçons d'un précepteur domestique, mais à condition que leurs parents y voient un quelconque intérêt, ce qui paraît toujours avoir été assez rare ; de plus, même avec le meilleur soutien familial, les lieux d'éducation supérieure (collèges, universités) étaient résolument fermés à toutes. Par ailleurs, d'innombrables hommes - qui avaient, eux, fréquenté ces lieux, et qui en tiraient de grands avantages sociaux et symboliques - déclinaient sur tous les registres les dangers de l'instruction pour les femmes et de l'existence des femmes instruites pour leurs familles, voire pour la société tout entière. Le courage et l'intelligence de celles qui s'éduquèrent par elles-mêmes ou consolidèrent leur instruction dans ce contexte hostile ${ }^{2}$, qui s'imposèrent dans les cercles intellectuels 
avec leurs propres écrits, et, davantage encore, qui se donnèrent les moyens de les voir publiés, n'en apparaissent que plus grands. Enfin, les seules femmes ayant un accès simplifié (sinon simple) à la culture et à l'édition, celles de l'aristocratie princière, étaient généralement détournées de la publication: dans la grande noblesse, il était bien vu de savoir écrire avec talent, mais certainement pas de mettre ses écrits en vente!

3 Là encore, le courage de celles qui transgressèrent cette injonction doit être souligné, d'autant qu'elles ouvrirent ainsi toutes grandes plusieurs portes aux autres femmes, qu'elles soient leurs jeunes homologues ou qu'elles appartiennent à d'autres milieux ${ }^{3}$, en démontrant que les femmes pouvaient s'exprimer publiquement, qu'elles pouvaient le faire dans tous les genres, et qu'elles pouvaient dénoncer le sort fait à leur sexe - ce que seule Christine de Pizan avait osé faire. En effet, si Marguerite d'Autriche fait paraître des vers de déploration qui s'inscrivent encore dans une certaine tradition "féminine » (Complainte de dame Marguerite, Anvers, 1492), Anne de France innove avec un traité d'éducation des princesses dans lequel elle dénonce le système de jugement des «deux poids, deux mesures» qui défavorise systématiquement les femmes (Enseignemens à sa fille..., Lyon, vers 1520). La suivante, Marguerite de Navarre, auteure de poésie religieuse, de théâtre, de contes..., fait publier plusieurs de ses œuvres à partir de 1531, et son Heptaméron (1559) est un véritable réquisitoire contre les violences que subissent les femmes. Quant à sa propre fille, Jeanne d'Albret, elle s'aventure encore un peu plus loin en rédigeant un long mémoire justificatif adressé à Catherine de Médicis et Charles IX, dans lequel elle explique ce qui l'a conduite à prendre les armes contre la couronne (Ample déclaration..., 15704).

4 Ces exemples, et notamment le dernier, durent jouer pour Marguerite de Valois. Son premier écrit connu est en effet un mémoire justificatif, postérieur de quatre ans à celui de Jeanne d'Albret - entre temps devenue sa belle-mère. Elle ne fit toutefois pour lors que prêter sa plume à son mari, le futur Henri IV, impliqué dans la conjuration dite des « Malcontents » et sommé de s'expliquer sur ses agissements. Nul ne semble avoir su, à l'époque, qu'il avait appelé son épouse à son aide : c'est sous le titre Déposition du roi de Navarre que ce court et vigoureux plaidoyer écrit à la première personne du masculin singulier fut publié dès 1578 , et c'est sous ce titre qu'il fut réédité deux siècles durant ${ }^{5}$. L'un des intérêts majeurs de ce texte, rédigé en quelques jours dans le vif des événements qui devaient mener La Mole et Coconat sur l'échafaud, est qu'il montre l'implication personnelle de la reine dans la conspiration, et la teneur des convictions qui liaient les conjurés. Les complots du printemps 1574 sont en effet la première manifestation du courant politique né du traumatisme de la Saint-Barthélemy, réuni autour de l'idée très embryonnaire encore de "déconfessionnalisation de l'État », et dont les efforts allaient aboutir à l'Édit de Nantes (1598). Une partie de la grande noblesse était persuadée que les divisions religieuses étaient orchestrées pour l'écarter des affaires, voire pour l'éliminer physiquement, d'où la présence dans cette coalition de nombreux princes et princesses (Marguerite, mais aussi Henriette de Clèves, duchesse de Nevers, Claude-Catherine de Clermont, maréchale de Retz...), et c'est cette manipulation que la reine dénonce à travers l'» autobiographie » du Béarnais ${ }^{6}$.

Il semble - au vu des textes qui demeurent - qu'elle ait ensuite rangé cette plume "politique» pour un bon moment. Elle développe, en revanche, une importante activité intellectuelle avec la maréchale de Retz, qui fréquente comme elle l'académie du Palais créée par son frère Henri III. Les deux amies sont aussi, avec Henriette et 
d'autres femmes (dont l'Hélène de Ronsard), les plus en vue des « neuf muses » du salon que la maréchale a ouvert à Paris dans les années 1570. Beaucoup d'écrivains, de traducteurs, d'érudits, d'artistes fréquentent ce cercle, cherchant et trouvant auprès de ces princesses des soutiens autant que des inspiratrices, comme en témoignent les œuvres qui leur sont dédiées ou qui les évoquent ${ }^{7}$. Dans les années qui suivent son départ de Paris et son installation en Gascogne auprès de son mari (1578), cette activité de mécène et de juge éclairé de la production artistique de son temps ne fait que se renforcer. C'est sans doute durant son voyage ou au début de son séjour à Nérac qu'elle demande à Brantôme d'écrire sa vie ("Vous me dites un jour que j'écrisse de vous... »), requête qui aboutira dans les années 1590 au fameux Discours sur la reine de France et de Navarre, Marguerite ${ }^{8}$. Installée dans la capitale des Albrets, elle en fait un foyer artistique où se retrouvent catholiques et huguenots, en accord avec les convictions évoquées plus haut. Tout ce que le sud-ouest compte alors de poètes participe à son Album de poésie ${ }^{9}$, elle fait aussi beaucoup jouer des musiciens, comme en témoignent ses comptes, et elle encourage les traductions : celle du Commentaire sur le Banquet de Platon de Marcile Ficin par Guy Le Febvre de La Boderie en 1578, celle du Pimandre de Mercure Trismégiste par François de Foix-Candale en 1581, celle de l'Aminta du Tasse par Pierre de Brach en 1584...

6 La fin de la décennie 1580 est pour Marguerite de Valois des plus malheureuse et mouvementée. Alors que la France s'enfonce dans la guerre civile, elle «abandonne " (selon ses propres termes) le roi de Navarre, et après plus d'une année de combats, de déboires et d'errance, elle trouve en Auvergne ce qu'elle appellera son "arche de salut »: la forteresse d'Usson, où elle passera près de vingt ans (1586-1605). Ce repos forcé la pousse, vers 1593-1594 - au moment où son mari devient roi de France -, à se mettre à l'écritoire, pour répondre au Discours que Brantôme a rédigé sur elle et lui a fait parvenir. Il en sortira les Mémoires de la reine Marguerite, le premier texte de ce genre qu'une femme ait écrit en France, et à bien des égards le premier tout court de ces " Mémoires aristocratiques » qui se multiplieront aux xviie et xviiie siècles, dans le sillon de son succès. L'ouvrage, publié en 1628 , connaîtra en effet une trentaine d'éditions et réimpressions durant le seul xviie siècle, et il sera l'unique livre d'une femme retenu en 1638 par les Académiciens parmi les trente les plus justement célèbres du temps.

7 Les deux décennies passées à Usson ne sont cependant pas entièrement dédiées à l'écriture de ce petit chef d'œuvre. Après les premières années, fort noires, consacrées à la simple survie, Marguerite a réorganisé autour d'elle une cour, embauché des musiciens, noué des relations étroites avec les lettrés de la région (les Urfé, Loys Papon...), attiré des intellectuels des deux sexes (notamment Madeleine de Senneterre $\mathrm{e}^{10} \ldots$ ), fait aménager un théâtre dans son château, et elle s'est remise à l'étude ${ }^{11}$. L'un de ses poèmes, adressé à Antoine La Pujade, montre que la philosophie, et en particulier l'ésotérisme, font alors toujours partie de ses préoccupations («Est-ce pour acquérir ce grand nom de Pimandre / qu'en silence et muet allez toujours rêvant $\left.{ }^{12} \ldots »\right)$. Ce texte témoigne en outre, au-delà de la familiarité de Marguerite avec «ses » auteurs, de sa volonté de s'insérer dans la communauté des créateurs, puisqu'il est publié dans les CEuvres chrestiennes du poète en 1604. Cette volonté se repère d'ailleurs dès 1599 avec l'insertion d'un autre poème, alors non signé, dans les Muses françoises ralliées, l'un de ces florilèges de poésies anonymes dont la mode se répand à partir de la fin du siècle, et derrière laquelle pourrait être Marguerite elle-même ; 
beaucoup de poètes publiés dans ces recueils sont en effet ceux de son cercle ou ceux, décédés, qu'elle a particulièrement aimés.

8 La reine, à présent « démariée » d'Henri IV (déc. 1599), prépare en effet sa rentrée à Paris - qui n'a cependant lieu qu'en 1605. À partir cette date, et durant la dizaine d'années qui la séparent de sa mort, elle fait office de ministre de la culture en même temps qu'elle est chargée par son ex-mari, puis par la régente Marie de Médicis, de certaines réceptions d'ambassadeurs. Installée dès 1607 sur la rive gauche de la Seine, en face du Louvre, dans un magnifique hôtel qu'elle a fait construire (et dont il ne reste aujourd'hui que la chapelle des Beaux-Arts), elle anime une cour qui est aussi le premier salon du siècle. Les intellectuels français, comme les étrangers de passage, viennent y converser avec une femme qui a tiré de ses années de réclusion une érudition étonnante, mais qui les impressionne par bien d'autres aspects encore. D'une part, on entend chez elle - y compris pendant les repas, car la reine entend que tous les plaisirs soient mêlés - des compositeurs encore inconnus qui ravissent l'auditoire ${ }^{13}$. D'autre part, on rencontre chez elle un nombre impressionnant de grands esprits (Vincent de Paul, Elie Pitard...), de philosophes (Scipion Dupleix, Jean de SaintFrançois...), et surtout de poètes (Desportes, Maynard, La Roque, Champeygnac, Garnier, Deimier, Marc de Mailliet, Alary, Vital d'Audiguier, Corbin, Régnier, Viau, Malherbe ${ }^{14} \ldots$ ), dont plusieurs travaillent pour elle, chantent ses maisons, ses actions, ses amis, ses amours. Son éclectisme lui permet de goûter leurs productions si différentes, et d'encourager aussi bien les créateurs débutants que les ceux qui sont en perte de vitesse, de soutenir aussi bien les œuvres légères que les sérieuses. Si elle n'est guère attirée par le nouveau purisme, elle continue en revanche de promouvoir très activement la pastorale ${ }^{15}$, et c'est chez elle que s'orchestrent les premiers succès de L'Astrée, de son ex-voisin et ami, le Forézien Honoré d'Urfé.

Celle qu'on appelle depuis son divorce la reine Marguerite fait ainsi (mais avec plus de moyens désormais) ce qu'elle a toujours fait, ce qu'avaient fait ses amies (la duchesse de Nevers avait commandité des traductions de l'Arioste), et ce qu'avaient fait avant elles une bonne partie des femmes de leur "caste ": Anne de Bretagne, surnommée "le refuge des savants hommes »; Renée de Ferrare, Marguerite de Navarre, Marguerite de Savoie, protectrices de tant d'intellectuels du siècle et mécènes pour tant d'artistes; ou encore sa propre mère, Catherine de Médicis, à l'origine de la fortune du théâtre italien en France, et par ailleurs l'une des grandes bâtisseuses de Paris.

Enfin, Marguerite réunit autour d'elle des intellectuelles et des créatrices, comme, là encore, la plupart de ses parentes. On sait ainsi que Marie de Gournay, future auteure de L'égalité des hommes et des femmes, a travaillé chez elle, de même que Mademoiselle de Choisy, qui tiendra plus tard un salon, de même qu'Antoinette de La Tour et Mademoiselle de Beaulieu, toutes deux poétesses. Certaines lui dédient leurs œuvres, toutes participent à l'émulation intellectuelle qui caractérise la cour de la dernière des Valois, et toutes paraissent le faire, avec elle, dans une optique féministe. «Les communs exercices de leur sexe sont tenus pour profanes, écrit ainsi un historien contemporain. Elle renvoie aux dames de ville les ouvrages et veut que leurs esprits s'élèvent plus haut, qu'ils courent dans les bons livres, s'arrêtent sur les bons exemples, et fassent passer heureusement le génie et les grâces d'une langue en une autre. La musique, la peinture, la poésie et les mathématiques ne permettent qu'une heure de temps leur échappe sans plaisir ou profit ${ }^{16}$. » On reconnaît ici l'appel lancé près de cinquante ans plus tôt par Louise Labé, qui implorait ses semblables à s'élever "par 
dessus leurs quenouilles et fuseaux " pour atteindre à la connaissance et goûter la félicité que donnent « les sciences ", c'est-à-dire le savoir ${ }^{17}$ - un appel entendu, réitéré, et presque devenu un lieu commun de la rhétorique féministe.

11 Au crépuscule de sa vie, toutefois, Marguerite entend faire davantage encore : écrire et publier sur le sujet, autrement dit s'inscrire dans la gigantesque polémique qui, depuis le début du xve siècle, mettait régulièrement aux prises partisans et détracteurs des femmes. Cette Querelle l'avait jusque là, semble-t-il, laissée indifférente, au contraire de la plupart des auteures de la Renaissance, et l'on comprend bien pourquoi à la lecture de ses œuvres: Marguerite refusait son identité de femme. Mais ce refus commença à se disloquer durant les pourparlers de son « divorce » avec Henri IV, et finit par se transformer en son contraire. Aussi la reine se lance-t-elle dans la polémique, un jour de 1614, après avoir lu un texte misogyne écrit par un jésuite... dont elle subventionnait la compagnie! Sortie victorieuse d'une joute oratoire publique avec lui, improvisée chez elle alors qu'il venait lui présenter son ouvrage, elle rédige après son départ une longue lettre résumant ses propos, que le Père insère aussitôt dans un livre sous presse, et à laquelle il donne le titre de Discours docte et subtil dicté promptement par la reine Marguerite ${ }^{18}$.

Le texte, extrêmement malicieux, n'a rien de la profondeur des analyses de Christine de Pizan ni de la force des dénonciations de Marguerite de Navarre ou d'Hélisenne de Crenne. Centré néanmoins sur une « question d'école » que traitait le jésuite (Pourquoi l'homme rend tant d'honneur à la femme ?), il se situe sur le terrain de la rhétorique scholastique - fleuron des lieux de savoir interdits aux femmes. Un terrain que Marguerite emprunte pour démontrer avec brio la supériorité du sexe dit faible sur l'autre, et qu'elle subvertit en dénonçant dans sa conclusion le fait que la recevabilité d'un argument n'est pas fonction de sa justesse, mais de la position d'autorité de qui le soutient (le Père, même s'il a tort, plutôt qu'elle, même si elle a raison). Ce message sera si parfaitement compris des contemporains de la reine, que le Discours sera réédité peu après sa mort par une féministe anonyme, en guise de réplique au tonitruant Alphabet de la malice des femmes, dédié à la plus mauvaise (1617), en annexe de son Excellence des femmes, avec leur réponse à l'autheur de l'Alphabet (1618).

Il apparaît ainsi que le mythe de la reine Margot, jeune princesse délurée multipliant les amants et leur portant malheur, qu'Alexandre Dumas inventa dans les années 1840, et auquel travaillèrent tant de ses successeurs (historiens ou romanciers), ne servit pas seulement à justifier l'exclusion des femmes du pouvoir perpétrée pendant la Révolution et maintenue par tous les régimes jusqu'en 1944. À l'évidence, il servit aussi à masquer comment de telles femmes avaient utilisé leur notoriété et leur savoir pour agir, seules ou avec d'autres, pour écrire, pour modifier le rapport de forces entre les sexes au profit de leurs semblables.

\section{BIBLIOGRAPHIE}

Berriot-Salvadore Evelyne, 1990, Les Femmes dans la société française de la Renaissance, Genève, Droz. 
,- 1994 , « Le temps des malheurs, le temps de la philosophie : Marguerite et la vulgarisation des sciences ", in Marguerite de France..., pp. 255-267.

Brantôme 1991, Recueil des Dames, poésies et tombeaux, éd. Etienne Vaucheret, Paris, Gallimard, collection « la pléiade ».

Broomhall Susan, 1998, «French Women in Print, 1488 to 1599 », Biblio-graphical Society of Australia and New Zealand Bulletin, 4, pp. 195-231.

Droz Eugénie, 1964, « La reine Marguerite de Navarre et la vie littéraire à la cour de Nérac, 1579-1582 », Bordeaux, Taffard, 1964 (Extrait du Bulletin de la société des bibliophiles de Guyenne 80, juillet-déc. 1964, pp. 77-120).

Gioanni Florence, 1996. La Société artistocratique française du XVI e siècle et la musique : le cas de Marguerite de Valois, Thèse de doctorat, Centre d'Etudes Supérieures de la Renaissance, Tours, 2 vol.

Kemp William, 1998, « Textes composés ou traduits par des femmes et imprimés en France avant 1550 : bibliographie des imprimés féminins (1488-1549) ", in L'Écriture des femmes à la Renaissance française, numéro spécial de Littératures, 18, Montréal, McGill [l'ensemble du Xvi siècle sera publié chez Champion].

Labé Louise, 1981, Euvres complètes, éd. Enzo Giudici, Genève, Droz.

Lavaud Jacques, 1936, Un Poète de cour au temps des derniers Valois, Philippe Desportes, 1546-1606, Paris, Droz.

Magnien-Simonin Catherine, 1994, «La jeune Marguerite des poètes », in Marguerite de France..., pp. 135-158.

Marguerite de France, reine de Navarre et son temps, 1994, Actes du colloque d'Agen [1991], Agen, Centre Matteo Bandello.

Marguerite de Valois 1998, Correspondance, 1569-1614, éd. Éliane Viennot, Champion.

-, 1999, Mémoires et autres écrits, 1574-1614, éd. Eliane Viennot, Paris, Champion.

Matthieu Pierre, 1631, Histoire de France, Paris, Veuve Nicolas Buon.

Ratel Simone, 1924, « La Cour de la reine Marguerite », Revue du Seizième Siècle, 11 (1924), pp. 1-29, 193-207 [suite 1925, même lieu, 12 (1925), pp. 1-43].

Viennot Éliane, 1993, Marguerite de Valois, histoire d'une femme, histoire d'un mythe, Paris, Payot.

Zilli Luigia, « Marguerite et la culture italienne », in Marguerite de France..., 239-254.

\section{NOTES}

1.Le premier écrit de femme publié semble en fait L'Art de chevalerie selon Vegece, de Christine de Pizan, mais il parut sans nom d'auteur. Voir Kemp 1998 ; en attendant la suite de sa bibliographie, voir, pour la période 1550-1610, Berriot-Salvadore 1990 : 542546 et Susan Broomhall 1998 : 195-231.

2.Montaigne donne un aperçu des deux phénomènes (et de la perversion des arguments opposés à la demande de savoir des femmes) dans l'un de ses Essais : « Quand je les vois attachées à la rhétorique, à la judiciaire [au droit], à la logique, et semblables 
drogueries si vaines et inutiles à leur besoin, j'entre en crainte que les hommes qui le leur conseillent le fassent pour avoir loi de les régenter sous ce titre. » (Livre III, ch. 3). 3.Marie d'Ennnetières dédicace ainsi son Epistre tresutile... (1539) à Marguerite de Navarre; voir également dernière note.

4.Texte connu sous le nom de Mémoires depuis sa réédition par de Ruble en 1893. 5.Mémoire justificatif pour Henri de Bourbon, in Marguerite de Valois 1999.

6.Les historiens n'ont presque jamais analysé cet épisode sous cet angle : l'échec des conjurés, leurs divisions, et peut-être surtout l'importante présence de princesses à leurs côtés, les ont conduit à n'y voir que confusion et en réalité, à le lire à travers les romans qui l'ont popularisé (Le Rouge et le noir, La Reine Margot, les Histoires d'amour de l'histoire de France...).

7.Voir Jacques Lavaud 1936 : 70-107 (pages consacrées au salon de la maréchale), et Catherine Magnien-Simonin $1994: 144$ et suiv.

8.Brantôme 1991 : 119-158; le poème est p. 906.

9.Voir Eugénie Droz 1964.

10.Ou Saint-Nectaire ; voir sa Correspondance 1998 : 444.

11.Voir Berriot-Salvadore 1994.

12. "Sonnet de la reine Marguerite sur les œuvres et silences d'Antoine la Pujade », Marguerite de Valois 1999 : 294-295. La plupart de la production poétique de la reine est perdue.

13.Monteverdi, qui travaille à la cour des Gonzague (cousins des Nevers), en faisait sans doute partie. Une thèse récemment consacrée à ce sujet (F. Gioanni 1996) n'a toutefois pas permis d'identifier quels compositeurs étaient joués chez Marguerite.

14. Voir Simone Ratel 1924, et Jean Balsamo 1994.

15. Voir sur ce point Luigia Zilli 1994.

16.Pierre Matthieu 1631 : vol. 2, p. 40.

17.Louise Labé 1981 : 17-20 (« A M.C.D.B.L [Dédicace à Clémence de Bourges] », 1555).

18.Marguerite de Valois 1999.

\section{RÉSUMÉS}

Bien qu'Alexandre Dumas l'ait représentée en train de donner des leçons (particulières) de grec au beau La Mole, Marguerite de Valois n'est plus connue pour être une intellectuelle. C'est l'une des évidences que le mythe de la Reine Margot a été chargé de recouvrir, avec un succès qu'on ne mesure pas puisque la réalité a disparu derrière lui. Sa vie montre en effet que, née dans un groupe social où les femmes étaient des mécènes, et à une époque où des femmes très diverses avaient décidé d'investir la scène littéraire, elle a utilisé sa notoriété, ses moyens et sa très grande culture pour promouvoir des idées, aussi bien sur le plan politique que philosophique et littéraire, aussi bien par l'encouragement à la création que par l'écriture de plusieurs œuvres et la publication de certaines. L'entreprise de distorsion et d'effacement de cette mémoire était sans doute d'autant plus nécessaire que, comme bien des femmes de son époque moins en vue qu'ellemême, elle avait œuvré pour un monde mixte, dans un temps où la plupart des intellectuels travaillaient à organiser et à approfondir la partition des sexes. 
Alexandre Dumas may well have depicted Marguerite giving (private) Greek lessons to the handsome La Mole, but historians have neglected the intellectual status of "Queen Margot». This is but one of the certainties that her myth has the task of covering up - so successfully that the real woman has all but disappeared. Women were patrons in Marguerite's social universe ; and hers was a moment when women of very different profiles chose to hoist themselves onto the literary stage. She used her fame, her wealth, and her extensive culture to promote a series of political, philosophical, and literary ideas; and she did this by supporting creativity and by writing (and sometimes publishing) herself. At a moment when most male intellectuals were constructing, and deepening, the gender divide, the distortion and erasing of her memory was no doubt especially necessary because, like so many less famous women of her age, she sought to foster a world of gender exchange.

\section{AUTEUR}

\section{ÉLIANE VIENNOT}

Éliane VIENNOT est agrégée de lettes modernes, professeure de littérature de la Renaissance à l'Université de Saint-Étienne, elle s'intéresse aux relations entre les femmes et la sphère publique. Une grande partie de son travail a été consacré à Marguerite de Valois (étude historique et mythocritique, édition de ses (Euvres complètes, nombreux articles). Elle a également publié Femmes et pouvoirs sous l'Ancien Régime (dir., avec D. Haase-Dubosc, Rivages, 1991), La Démocratie 'à la française' ou les femmes indésirables (dir., P.U. ParisVII, 1996), Royaume de Fémynie. Pouvoirs, contraintes, espaces de liberté des femmes de la Renaissance à la Fronde (dir., avec Kathleen Wilson-Chevalier, Champion, 1999). Elle travaille actuellement sur une histoire de la loi salique. 